\title{
Erratum to: Distortion of price discount perceptions through the left-digit effect
}

\section{Chien-Huang Lin ${ }^{1} \cdot$ Jyh-Wen Wang $^{1}$}

Published online: 15 June 2016

(C) Springer Science+Business Media New York 2016

\section{Erratum to: Mark Lett (2015) \\ DOI 10.1007/s11002-015-9387-5}

The original version of this article unfortunately contained an error in the wording on page 4 line 9, "For example, because 4 is $33 \%$ greater than 3 , and 9 is $13 \%$ greater than 8 , the absolute difference between 3 and 4 is perceived to be greater than the difference between 8 and 9". The word "absolute" should be "relative".

The online version of the original article can be found at http://dx.doi.org/10.1007/s11002-015-9387-5.

Jyh-Wen Wang

ldreamertw@msn.com; m8933014@msg.ndhu.edu.tw

1 Department of Business Administration, National Central University, No. 300, Jhongda Rd., Jhongli City, Taoyuan County 32001, Taiwan 